\title{
National Early Warning Score (NEWS) as Prognostic Triage Tool for Septic Patients
}

This article was published in the following Dove Press journal:

Infection and Drug Resistance

\begin{abstract}
Abdulaziz Almutary
Saqer Althunayyan $\mathbb{D}^{2}$

Khaled Alenazi ${ }^{3}$

Abdulrahman Alqahtani ${ }^{4}$

Badar Alotaibi ${ }^{5}$

Marwa Ahmed ${ }^{6}$

Isam S Osman ${ }^{7}$

Adil Kakpuri ${ }^{8}$

Abdulaziz Alanazi ${ }^{9}$

Mohammed Arafat ${ }^{10}{ }^{10}$

Abdulmajeed Al-Mutairi ${ }^{\prime \prime}$

Fatma Bashraheel $\mathbb{D}^{12}$

Faisal Almazroua ${ }^{1}{ }^{1}$

'Emergency Department, King Saud Medical City, Riyadh, Saudi Arabia; ${ }^{2}$ Department of Accident and Trauma, Prince Sultan Bin Abdulaziz College for Emergency Medical Services, King Saud University, Riyadh, Saudi Arabia; ${ }^{3}$ Internal Medicine Department, King Saud Medical City, Riyadh, Saudi Arabia; ${ }^{4}$ Vision Realization Office, Ministry of Health, Riyadh, Saudi Arabia; ${ }^{5}$ Emergency Department, Riyadh Care Hospital, Riyadh, Saudi Arabia; ${ }^{6}$ Pharmaceutical Care Services Department, King Saud Medical City, Riyadh, Saudi Arabia; ${ }^{7}$ Vascular Surgery

Department, King Saud Medical City, Riyadh, Saudi Arabia; ${ }^{8}$ Quality Management and Patient Safety Administration, King Saud Medical City, Riyadh, Saudia Arabia;

${ }^{9}$ Nursing Administration, King Saud Medical City, Riyadh, Saudia Arabia; ${ }^{10}$ Emergency Medicine Department, King Saud University Medical City, Riyadh, Saudi Arabia; " 'College of Medicine, King Saud University, Riyadh, Saudia Arabia; ${ }^{12}$ College of Medicine, Alma'arefa University, Riyadh, Saudi Arabia
\end{abstract}

Correspondence: Saqer Althunayyan Department of Accident and Trauma, Prince Sultan Bin Abdulaziz College for Emergency Medical Services, King Saud University, Riyadh, Saudi Arabia

Email salthunayyan@ksu.edu.sa
Purpose: We assessed the National Early Warning Score (NEWS) in emergency triage for predicting sepsis-related outcomes.

Methods: A retrospective chart review of all cases enrolled in the sepsis management protocol for a one-year duration. The protocol utilized the NEWS as a screening tool for sepsis in the triage area. Primary outcomes of interest were hyperlactatemia, admission to ICU and intrahospital mortality. Sensitivity, specificity, and area under the curve (AUC) were calculated for a given NEWS.

Results: A total of 444 patients were reviewed from July 2018 to June 2019, with a mean age of 58.7 years. A NEWS $\geq 5$ was more than $88 \%$ sensitive in predicting hyperlactatemia, ICU admission, and/or mortality. Specificity, on the other hand, was as low as $12 \%$. The AUC for the NEWS was 0.667 for predicting hyperlactatemia and 0.602 for predicting ICU admission or mortality.

Conclusion: The NEWS was a sensitive screening tool for predicting sepsis-related outcomes. However, it was not specific, and further studies are recommended to assess the integration of other factors to improve specificity.

Keywords: sepsis, lactate, predictor, mortality, septic shock

\section{Introduction}

Sepsis is a life-threatening disease that requires early recognition and immediate management. A local study conducted at the National Guard Hospital in Riyadh, Saudi Arabia, showed that the mortality rates for sepsis and septic shock were higher than the reported global mortality rates approaching $60 \%{ }^{1}$. Internationally, the incidence and severity of sepsis reveal a rising pattern ${ }^{2,3}$. Several studies emphasize that early recognition and management of sepsis can avert its progression and decrease the associated mortality, morbidity and financial burden. ${ }^{4-6}$ Therefore, the Surviving Sepsis Campaign 2018 considered the time of emergency triage as 'time zero' for identification and starting the hour-1 bundle of management. ${ }^{7}$

However, identification of the septic patient is not always clinically straightforward. The signs of sepsis are often subtle, nonspecific, and frequently missed in an emergency triage or prehospital setting. ${ }^{8-10}$ The traditional systemic inflammatory response syndrome (SIRS) criteria rely on laboratory results, such as white blood cells, neutrophil counts, and Pco2, which make it unsuitable for use in triage. As such, there is an urgent need for a screening tool that has the ability to identify sepsis at triage.

A few emergency department (ED) triage tools have been suggested to risk stratify septic patients and predict their outcomes. One such commonly used tool is the quick Sepsis-related Organ Failure Assessment (qSOFA). It is a simplified version of the 
well-validated SOFA tool used in the intensive care unit (ICU) and research. It depends on three parameters: level of consciousness, respiratory rate, and systolic blood pressure. While qSOFA is a good prognostic tool in the ICU literature, it is less sensitive in predicting sepsis-related adverse outcomes if used as an emergency triage tool. ${ }^{11-13}$ However, a screening tool must exhibit high sensitivity. The National Early Warning Score (NEWS) was first introduced by the Royal College of Physicians in 2012 as a predictor of patient deterioration. However, it was not specifically designed for septic patients. It includes seven parameters (temperature, systolic blood pressure, respiratory rate, oxygen saturation, oxygen supply, heart rate, and level of consciousness) as shown in (Table 1). The score range is from 0 to 20. Patients are classified as a low score (NEWS 1-4), medium score (NEWS of 5-6) and high score (NEWS $\geq 7$ ). ${ }^{14}$

A large observational study conducted for all septic patients in an ED revealed an association between elevated NEWS, ICU admission, and mortality. ${ }^{15}$ Subsequently, two large retrospective ED studies of patients with suspected sepsis revealed that the NEWS was more accurate than qSOFA and SIRS in predicting ICU admission and mortality. ${ }^{16,17}$ Hence, the NEWS has started to emerge as a promising tool that may be utilized in the assessment and risk stratification of patients suspected of having sepsis in the triage area. Nonetheless, it is still not fully validated, and there is no conclusive evidence to endorse it in this clinical area. Furthermore, the NEWS has not yet been studied in a Saudi population for either the diagnosis of sepsis or as a prognostic tool for sepsis.

Therefore, the aim of this study is to assess the utility of the NEWS as a screening tool in adult patients with suspected sepsis at ED triage as a predictor of sepsis-related outcomes, such as hyperlactatemia, ICU admission and inhospital mortality. Hyperlactatemia as a marker of severe sepsis and surrogate indicator of poor prognosis. ${ }^{18,19 .}$

\section{Materials and Methods}

The study was conducted in the ED of King Saud Medical City (KSMC), Riyadh, Saudi Arabia. KSMC is a teaching hospital with an average of 500 emergency room visits daily. The files reviewed included cases between July 2018 until June 2019. The Ethical Committee of KSMC approved the study protocol with IRB approval number (H1RI - 28 - Jul19-02). All data were kept confidential throughout the study, and no data were shared with any third party and waiver of informed consent was granted from IRB in compliance with the declaration of Helsinki.

In our ED, in any patient presenting with suspected sepsis, the triage nurse calculated the NEWS. With a NEWS $\geq 5$, the patient was triaged to high acuity area and enrolled in the sepsis management protocol per KSMC sepsis management guidelines. In addition, the patient could be enrolled in a sepsis management protocol per the discretion of the treating ED physician, regardless of the NEWS at triage. Retrospectively, we reviewed all sepsis management protocol sheets. The exclusion criteria were age $<18$ years old, pregnancy, alternative diagnosis, or leaving the ED against medical advice.

The NEWS score, demographic features, comorbidities, lactate level, and ICU admission data were retrieved from KSMC's sepsis management protocol sheet. Mortality status and any other missed data in the management protocol sheet were retrieved from medical records. The sepsis management protocol of KSMC utilized the NEWS as a screening tool to detect patients with potential diagnoses of sepsis in the triage area of the ED. A data collector collated these data on a specific Cloud-based spreadsheet. To ensure the quality of the data collection process, a second investigator independently monitored this process online to check for missing data. Cases of discrepancy or missing data were arbitrated by a third reviewer.

Table I National Early Warning Score (NEWS)

\begin{tabular}{|c|c|c|c|c|c|c|c|}
\hline Physiological Parameters & 3 & 2 & $\mathbf{I}$ & 0 & $\mathbf{I}$ & 2 & 3 \\
\hline Respiration Rate (BPM) & $\leq 8$ & & $9-11$ & $12-20$ & & $21-24$ & $\geq 25$ \\
\hline Oxygen Saturation (\%) & $\leq 91$ & $92-93$ & $94-95$ & $\geq 96$ & & & \\
\hline Any Supplemental Oxygen & & Yes & & No & & & \\
\hline Temperature & $\leq 35$ & & $35.1-36.0$ & $36.1-38.0$ & $38.1-39.0$ & $\geq 39.1$ & \\
\hline Systolic Blood Pressure $(\mathrm{mmHg})$ & $\leq 90$ & $19-100$ & $101-110$ & $111-219$ & & & $\geq 220$ \\
\hline Heart Rate (BPM) & $\leq 40$ & & $4 I-50$ & $51-90$ & $91-110$ & $111-130$ & $\geq|3|$ \\
\hline Level of Consciousness & & & & Alert & & & $\mathrm{U}, \mathrm{P}$ or $\mathrm{V}^{*}$ \\
\hline
\end{tabular}

Note: *Unresponsive, react to pain, or loud voice. 
Patients enrolled in sepsis management protocol $(n=495)$

Excluded $(\mathrm{n}=51)$

- Pregnant $(n=5)$

- $\quad$ Age $<18(\mathrm{n}=16)$

- Discharged against medical advice $(n=11)$

- Alternative diagnosis $(n=19)$

*Asthma exacerbation $(\mathrm{n}=11)$

*Paroxysmal supraventricular tachycardia $(\mathrm{n}=1)$

* Hepatic encephalopathy $(\mathrm{n}=1)$

*Diabetic ketoacidosis $(\mathrm{n}=6)$

Patients enrolled per inclusion $(n=444)$

Figure I Flowchart demonstrating the inclusion and exclusion criteria.

Data were analyzed using STATA version 15.1 (StataCorp, College Station, TX, USA). Data normality was assessed by the Shapiro-Wilk test, continuous variables were described as the means and standard deviations, and categorical variables were described as numbers and percentages. The difference between groups was analyzed using a two-tailed Student's $t$-test for continuous variables in variables normally distributed and the chi-square test for categorical variables. For variables that did not comply with normal distribution, the median and interquartile range (IQR) were presented, and the Mann-Whitney $U$-test was used to analyze intergroup differences. Receiver operating characteristic (ROC) analysis was conducted to calculate sensitivity, specificity, positive predictive value (PPV), negative predictive value (NPV) and area under the curve (AUC) for the NEWS for each endpoint. The optimal cut-off value of the NEWS was measured and plotted for each outcome using Youden's index. A complete case analysis was performed, and statistical significance was determined at a $\mathrm{p}$ value less than 0.05 in all parts of the analysis.

The endpoints were hyperlactatemia (lactate equal or more than $4 \mathrm{mmol} / \mathrm{L})$; ICU admission; and in-hospital mortality. The lactate level was based on the initial serum laboratory result in the ED.

\section{Result}

Out of 495 patients enrolled in sepsis management protocol by the triage nurses only 444 subjects met our inclusion and exclusion criteria (Figure 1).

The mean age was 58.7 years, and $48.2 \%$ were male. Of these, 186 (41.9\%) patients were admitted to the ICU as reported in (Table 2). Pneumonia was by far the most frequent identified source of infection (40\%). Patients admitted to the ICU had a significantly higher lactate level $(p<0.05)$ and WBC count $(p=0.0438)$ than those admitted to non-ICU wards (Table 2). The lowest NEWS in our sample was 4, whereas the highest NEWS was 13. A history of hypertension was significantly greater in patients admitted to the ICU than those without hypertension $(\mathrm{p}=0.010)$. Out of 444 subjects, $127(28 \%)$ patients died, with $77 \%$ of these patients being admitted to the ICU. There was a statistically

Table 2 Patient Characteristic Among the Outcomes

\begin{tabular}{|l|l|l|l|l|}
\hline Characteristics & All Patients (N = 444) & $\begin{array}{l}\text { Not Admitted to } \\
\text { ICU (N = 258) }\end{array}$ & Admitted to ICU (N = I86) & p-value \\
\hline Age, mean (SD), years & $58.7(23.3)$ & $59.6(22.8)$ & $57.3(24.1)$ & 0.4113 \\
Male gender & $214(48.2)$ & $129(60.2)$ & $85(39.7)$ & 0.971 \\
Hypertension, N (\%) & $176(39.6)$ & $110(62.5)$ & $66(37.5)$ & 0.010 \\
DM & $158(40.1)$ & $96(60.7)$ & $62(39.2)$ & $11(32.4)$ \\
Chronic renal failure & $34(7.7)$ & $23(67.6)$ & $6(40.0)$ & 0.742 \\
Chronic heart failure, N (\%) & $15(3.4)$ & $9(60.0)$ & $88(50.0)$ & 0.898 \\
Pneumonia, N (\%) & $176(39.6)$ & $88(50.0)$ & $11(28.9)$ & 0.740 \\
Urinary tract infection, N (\%) & $38(9.0)$ & $27(71.0)$ & $86(51.8)$ & 0.617 \\
Other infection, N (\%) & $166(37.4)$ & $80(48.2)$ & $3.93(3.40)$ & $14.3(8.73)$ \\
Overall lactate, mean (SD) & $3.3(2.8)$ & $2.82(1.96)$ & $8.35(3.22)$ & 0.012 \\
Overall WBC, mean (SD) & $13.0(7.9)$ & $7.43(2.86)$ & $58(100.0)$ & 0.280 \\
NEWS & $7.85(3.07)$ & $0(00.0)$ & $98(77.2)$ & $<0.001$ \\
Required inotrope or vasopressor, N (\%) & $58(13.1)$ & $29(22.8)$ & 0.0097 \\
Mortality, N (\%) & $127(28.6)$ & & 0.0021 \\
\hline
\end{tabular}


Table 3 NEWS as a Predictor for Hyperlactatemia

\begin{tabular}{|l|l|l|l|l|l|}
\hline NEWS & Sensitivity, \% & Specificity, \% & NPV, \% & PPV, \% & AUC \\
\hline$\geq 4$ & $97.1(91.8,99.4)$ & $7.78(5.15,11.2)$ & $89.7(72.6,97.8)$ & $24.7(20.6,29.2)$ & $0.524(0.503,0.546)$ \\
$\geq 5$ & $95.2(89.2,98.4)$ & $13.8(10.3,17.9)$ & $90.2(78.6,96.7)$ & $25.6(21.3,30.2)$ & $0.545(0.517,0.573)$ \\
$\geq 6$ & $88.5(80.7,93.9)$ & $24.9(20.3,29.8)$ & $87.4(79,93.3)$ & $26.8(22.2,31.8)$ & $0.567(0.528,0.605)$ \\
$\geq 7$ & $79.8(70.8,87)$ & $39.8(34.5,45.3)$ & $86.4(79.9,91.4)$ & $29.2(24,34.9)$ & $0.598(0.551,0.645)$ \\
$\geq 8$ & $71.2(61.4,79.6)$ & $55.4(49.9,60.8)$ & $86(80.7,90.4)$ & $33.2(27,39.8)$ & $0.633(0.581,0.684)$ \\
$\geq 9$ & $59.6(49.5,69.1)$ & $65.9(60.5,70.9)$ & $84(79,88.2)$ & $35.2(28.2,42.8)$ & $0.627(0.574,0.681)$ \\
$\geq 10$ & $47.1(37.2,57.2)$ & $75.1(70.2,79.7)$ & $82(77.3,86.2)$ & $37.1(28.9,46)$ & $0.611(0.558,0.665)$ \\
$\geq 11$ & $34.6(25.6,44.6)$ & $83.5(79.1,87.3)$ & $80.4(75.8,84.4)$ & $39.6(29.5,50.4)$ & $0.591(0.541,0.641)$ \\
$\geq 12$ & $23.1(15.4,32.4)$ & $92.2(88.8,94.9)$ & $79.4(75,83.3)$ & $48(33.7,62.6)$ & $0.576(0.533,0.62)$ \\
$\geq 13$ & $19.2(12.2,28.1)$ & $95.2(92.3,97.2)$ & $79.1(74.8,83)$ & $55.6(38.1,72.1)$ & $0.572(0.532,0.612)$ \\
\hline
\end{tabular}

significant increase in the average NEWS in patients admitted to the ICU as compared to those admitted to non-ICU wards (8.35 [3.21] vs 7.51 [2.92], respectively, $\mathrm{p}<0.0058$ ).

NEWS $\geq 5$ had a sensitivity of $95.2 \%(89.2,98.4 \%)$ and specificity of $13.8 \%(10.3,17.9 \%)$ for predicting the hyperlactatemia (Table 3). The AUC of the NEWS in predicting hyperlactatemia was 0.6674 (95\% CI 0.6074 to 0.7273 ) (Figure 2). As the NEWS cut-off point increases, the sensitivity decreases and specificity increases as depicted in Table 3 and Figure 2. The optimal cut-off value for the NEWS to predict hyperlactatemia was 7.5, with a Youden index (J) of 0.265. Sensitivity at this cut-off point was $71 \%$ and specificity was $55 \%$.
NEWS $\geq 5$ was $93.9 \%$ (88.6, 96.2\%) sensitive and 6.10 $(3.29,10.20) \%$ specific for predicting ICU admission (Table 4). The AUC of the NEWS in predicting ICU admission was 0.591 (Figure 3).

When using a score of 5 as a threshold of positive NEWS screening, the sensitivity to predict mortality was as high as $89 \%$, while the specificity was as low as $11.9 \%$ as illustrated in Table 5 and Figure 4. The evaluation of the NEWS and mortality showed that the optimal cut-off value was also 7.5 , with a sensitivity of $62 \%$ and specificity of $54 \%$. When combining unfavorable outcomes (ICU and/or mortality), we obtained a similar sensitivity and specificity profile, $88.5 \%$ and $11.8 \%$, respectively (Table 6). The NEWS ROC analysis for combined worse outcome (ICU admission or mortality) is presented in Figure 5. The best

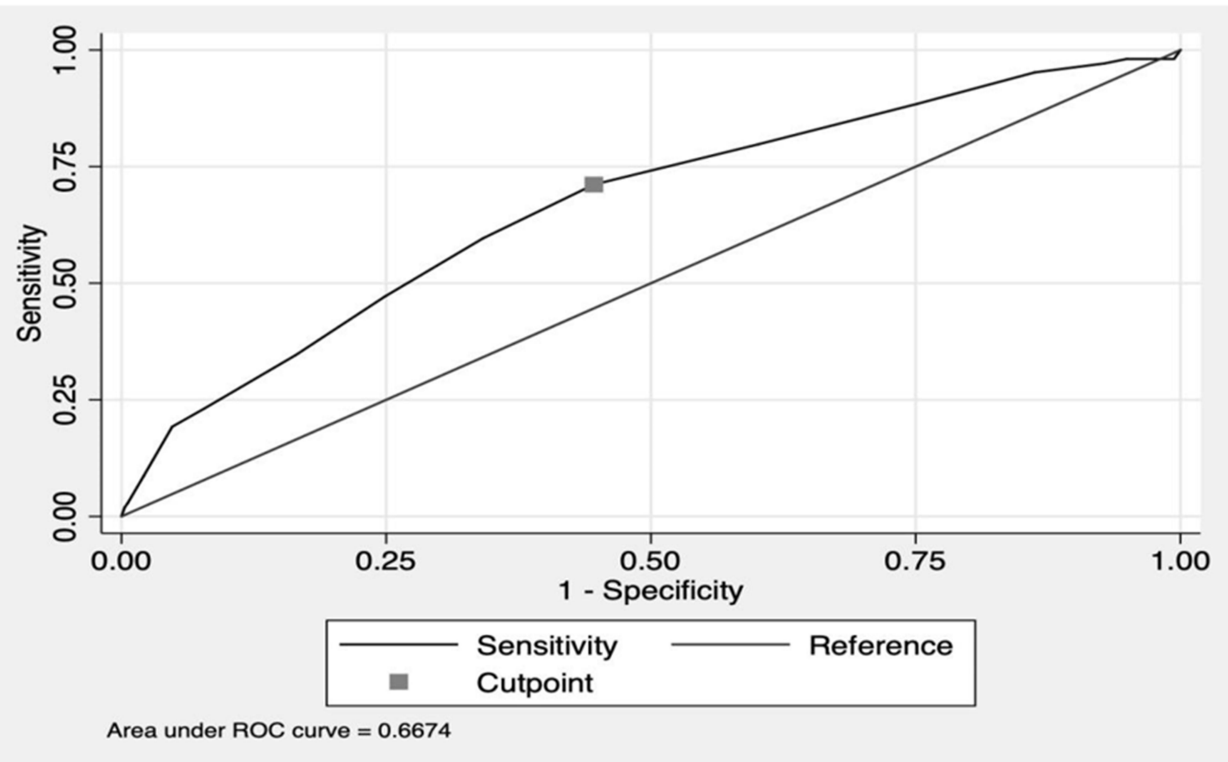

Figure 2 NEWS as a predictor for hyperlactatemia. 
Table 4 NEWS Characteristics for Prediction of ICU Admission

\begin{tabular}{|l|l|l|l|l|l|}
\hline NEWS & Sensitivity, \% & Specificity, \% & NPV, \% & PPV, \% & AUC \\
\hline$\geq 4$ & $93.9(88.6,96.2)$ & $6.10(3.29,10.20)$ & $48.1(28.7,68.1)$ & $48.5(43.4,53.6)$ & $0.495(0.472,0.520)$ \\
$\geq 5$ & $88.1(82.8,92.2)$ & $11.7(7.74,16.380)$ & $51.0(36.3,65.6)$ & $48.6(43.4,53.9)$ & $0.499(0.468,0.530)$ \\
$\geq 6$ & $82.2(76.2,87.2)$ & $24.9(19.2,31.2)$ & $59.6(48.6,69.8)$ & $50.9(45.4,56.5)$ & $0.535(0.496,0.575)$ \\
$\geq 7$ & $73.3(66.6,79.2)$ & $41.8(35.1,48.7)$ & $62.2(53.8,70.2)$ & $54.4(48.3,60.4)$ & $0.575(0.530,0.620)$ \\
$\geq 8$ & $58.9(51.8,65.8)$ & $55.4(48.5,62.2)$ & $58.7(51.6,65.6)$ & $55.6(48.7,62.4)$ & $0.572(0.524,0.619)$ \\
$\geq 9$ & $48.0(41.0,55.1)$ & $66.2(59.4,72.5)$ & $57.3(50.9,63.6)$ & $57.4(49.6,65.0)$ & $0.571(0.524,0.618)$ \\
$\geq 10$ & $37.6(30.9,44.7)$ & $76.1(69.7,81.6)$ & $56.3(50.3,62.1)$ & $59.8(50.8,68.4)$ & $0.568(0.524,0.613)$ \\
$\geq 11$ & $25.7(19.9,32.3)$ & $83.1(77.4,87.9)$ & $54.1(48.6,59.6)$ & $59.1(48.1,69.5)$ & $0.544(0.505,0.584)$ \\
$\geq 12$ & $15.8(11.1,21.6)$ & $92.5(88.1,95.6)$ & $53.7(48.4,58.9)$ & $66.7(51.6,79.6)$ & $0.542(0.511,0.572)$ \\
$\geq 13$ & $10.9(6.9,16.0)$ & $94.4(90.4,97.1)$ & $52.8(47.6,57.9)$ & $64.7(46.7,80.3)$ & $0.526(.5,0.553)$ \\
\hline
\end{tabular}

NEWS cut-off to predict ICU admission and/or mortality was 7.5 (Youden's index 0.192).

\section{Discussion}

This is the first study to predict ICU admission and mortality among patients with suspected sepsis using the NEWS at ED triage. The calculation of the NEWS by triage nurses has additive value, reflecting the practicality. The results demonstrated that a NEWS $\geq 5$ had a sensitivity of more than $88 \%$ for predicting hyperlactatemia or ICU admission or mortality; however, it was not specific to predict any of these outcomes. While a NEWS $\geq 7$ had a specificity of approximately $40 \%$ for hyperlactatemia or ICU admission or mortality, its sensitivity did not exceed $80 \%$.

Corfield et al conducted a retrospective study of patients visiting the ED with suspected sepsis. Their study revealed an association between the NEWS and patient mortality (odds ratio 1.95 to 5.64 ). ${ }^{15}$ Additionally, they found that a NEWS $\geq 5$ was $88 \%$ sensitive and $29 \%$ specific for predicting ICU admission and/or mortality. Although the sensitivity and specificity of a NEWS $\geq 7$ were $72 \%$ and $54 \%$, respectively, which is approximately consistent with our findings.

Moreover, Goulden et al conducted another large retrospective cohort study of 1818 adult patients in whom sepsis was suspected and admitted. ${ }^{20}$ Their study aimed to assess the NEWS as a predictor for ICU admission or mortality. They found that a NEWS $\geq 5$ had a sensitivity of $77 \%$ for predicting ICU admission and $74 \%$ for predicting mortality, while the specificity for them both was approximately $42 \%$. Their analysis revealed that a NEWS $\geq 7$ was the optimal threshold predicting mortality (sensitivity $56 \%$, specificity $67 \%$ ). Compared to our results, we found the NEWS was more sensitive for predicting these outcomes, but less

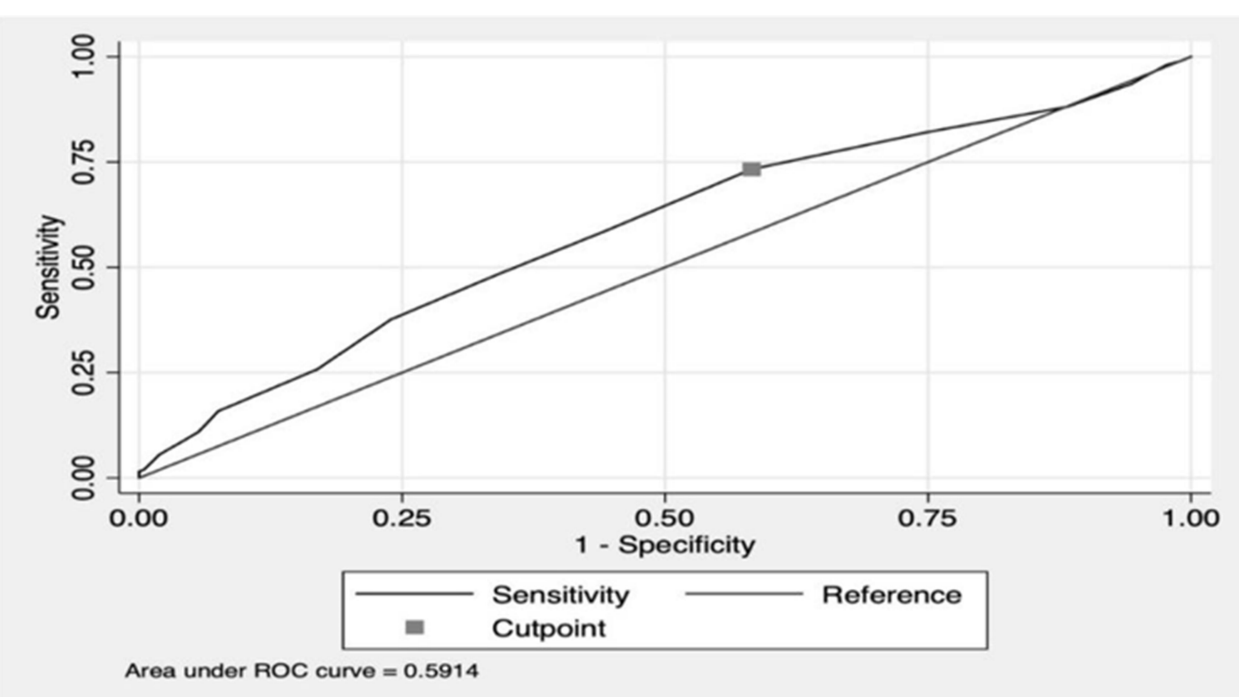

Figure 3 NEWS for prediction of ICU admission. 
Table 5 NEWS as a Predictor for Mortality

\begin{tabular}{|l|l|l|l|l|l|}
\hline NEWS & Sensitivity, \% & Specificity, \% & NPV, \% & PPV, \% & AUC \\
\hline$\geq 4$ & $93.7(88,97.2)$ & $6.75(4.23,10.1)$ & $72.4(52.8,87.3)$ & $29.1(24.7,33.8)$ & $0.502(0.477,0.528)$ \\
$\geq 5$ & $89(82.2,93.8)$ & $11.9(8.52,16)$ & $72.5(58.3,84.1)$ & $29.2(24.7,34)$ & $0.504(0.472,0.537)$ \\
$\geq 6$ & $84.3(76.7,90.1)$ & $24.1(19.5,29.3)$ & $78.9(69.4,86.6)$ & $31.2(26.3,36.4)$ & $0.542(0.502,0.582)$ \\
$\geq 7$ & $73.2(64.6,80.7)$ & $38.6(33.1,44.2)$ & $77.9(70.5,84.2)$ & $32.7(27.3,38.5)$ & $0.559(0.512,0.606)$ \\
$\geq 8$ & $62.2(53.2,70.7)$ & $53.7(48,59.3)$ & $77.7(71.5,83.1)$ & $35.4(29.2,42.1)$ & $0.58(0.529,0.63)$ \\
$\geq 9$ & $50.4(41.4,59.4)$ & $64(58.4,69.3)$ & $76(70.3,81)$ & $36.4(29.3,43.9)$ & $0.572(0.521,0.623)$ \\
$\geq 10$ & $40.2(31.6,49.2)$ & $74(68.7,78.7)$ & $75.2(69.9,79.9)$ & $38.6(30.3,47.5)$ & $0.571(0.521,0.62)$ \\
$\geq 11$ & $29.9(22.1,38.7)$ & $83(78.3,87)$ & $74.4(69.4,78.9)$ & $41.8(31.5,52.6)$ & $0.564(0.519,0.61)$ \\
$\geq 12$ & $18.9(12.5,26.8)$ & $91.6(88,94.5)$ & $73.5(68.8,77.8)$ & $48(33.7,62.6)$ & $0.553(0.515,0.59)$ \\
$\geq 13$ & $12.6(7.38,19.7)$ & $93.6(90.2,96)$ & $72.4(67.7,76.7)$ & $44.4(27.9,61.9)$ & $0.531(0.499,0.563)$ \\
\hline
\end{tabular}

specific. This inconsistency might be attributed to the inclusion criteria, which in our study provide a wider spectrum of severity as we considered all ED triage patients.

An additional retrospective cohort study compared the NEWS, qSOFA and SIRS of suspected septic patients in the ED with 30-day mortality. ${ }^{16}$ They found that a NEWS $\geq 5$ was $83 \%$ sensitive and $42 \%$ specific, whereas a NEWS $\geq 7$ was $68 \%$ sensitive and $66 \%$ specific. The AUC for the NEWS was 0.779 . They concluded that the NEWS was more accurate in predicting mortality than the qSOFA and SIRS.

Regarding the qSOFA as a predictor of sepsis-related outcomes, the reported sensitivity ranged from $31 \%$ to $47.6 \%$ for predicting ICU admission and 33-60\% for predicting mortality ${ }^{12,16,18,21-23}$. While the specificity for predicting ICU admission or mortality ranged $77-93.7 \%$ and the AUC ranged $0.58-0.74 .{ }^{12,16,18,21-23}$ A NEWS 5-7 was more sensitive than the qSOFA in predicting sepsis-related outcomes, but less specific. Overall, the predictive value of the qSOFA comes from its specificity. However, relying on poorly sensitive tools may endanger the patients and lead to high rate of missed cases of a preventable time-sensitive disease.

Our study supports the growing evidence that suggests the NEWS as a sensitive screening tool in the ED. It is a useful tool to assess patients with suspected sepsis in the triage setting. According to our results, a triage patient with a NEWS $<5$ indicates there is a $95 \%$ chance that the patient will not have hyperlactatemia and around an $89 \%$ chance that the patient will not require ICU admission or wind up deceased. On the other hand, a triage patient with a NEWS $\geq 7$ indicates there is a $40 \%$ chance of having hyperlactatemia, ICU admission or death. In such clinical scenarios, an argument can be made to require senior ED clinicians to assess patients in the triage area.

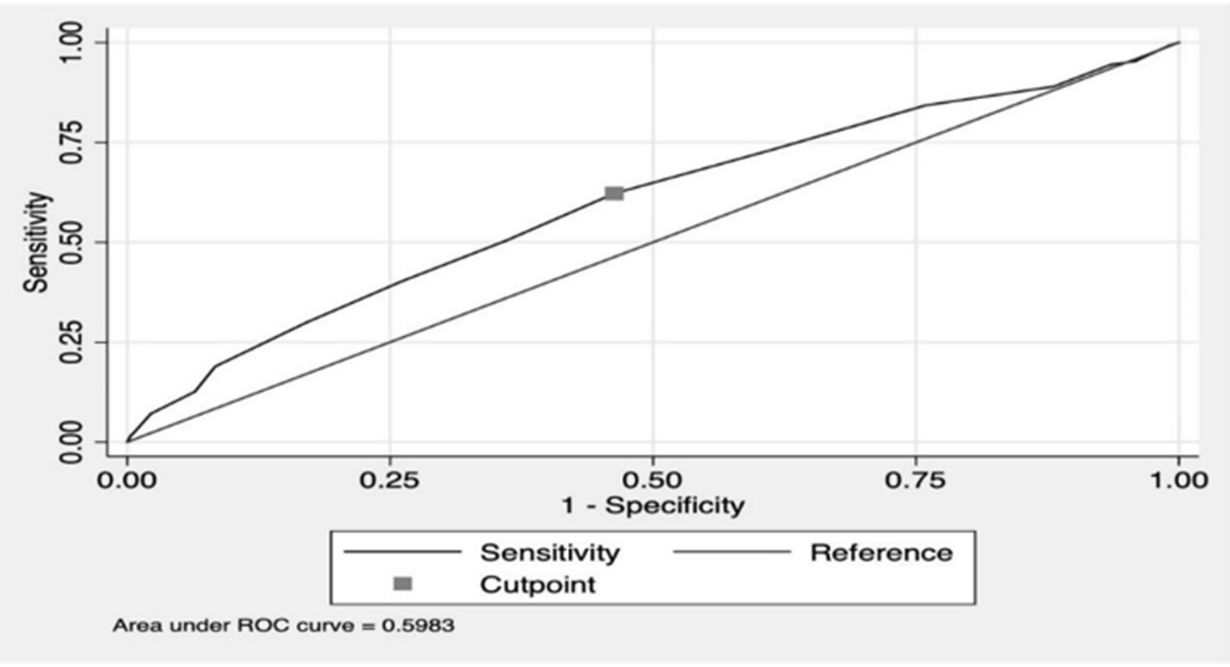

Figure 4 NEWS as a predictor of mortality. 
Table 6 NEWS as a Predictor for Combined Worse Outcome (ICU Admission or Mortality)

\begin{tabular}{|l|l|l|l|l|l|}
\hline NEWS & Sensitivity, \% & Specificity, \% & NPV, \% & PPV, \% & AUC \\
\hline$\geq 4$ & $93.6(89.6,96.4)$ & $6.86(3.80,11.2)$ & $48.3(29.4,67.5)$ & $53.5(48.6,58.5)$ & $0.502(0.479,0.526)$ \\
$\geq 5$ & $88.5(83.7,92.3)$ & $11.8(7.69,17.0)$ & $47.1(32.9,61.5)$ & $53.5(48.4,58.5)$ & $0.501(0.471,0.531)$ \\
$\geq 6$ & $82.5(77.0,87.1)$ & $26.5(20.6,33.1)$ & $56.8(46.3,67.0)$ & $56.3(50.8,61.6)$ & $0.545(.506,0.584)$ \\
$\geq 7$ & $72.6(66.5,78.3)$ & $44.1(37.2,51.2)$ & $58.4(50.2,66.3)$ & $59.9(53.9,65.6)$ & $0.584(0.539,0.628)$ \\
$\geq 8$ & $59.4(52.8,65.8)$ & $58.8(51.7,65.6)$ & $55.8(48.9,62.6)$ & $62.3(55.6,68.7)$ & $0.591(0.545,0.637)$ \\
$\geq 9$ & $47.9(41.3,54.5)$ & $68.6(61.8,74.9)$ & $53.4(47.2,59.6)$ & $63.6(56.1,70.7)$ & $0.582(0.537,0.628)$ \\
$\geq 10$ & $36.8(30.6,43.3)$ & $77.5(71.1,83.0)$ & $51.6(45.9,57.4)$ & $65.2(56.4,73.2)$ & $0.571(0.529,0.613)$ \\
$\geq 11$ & $24.8(19.4,30.8)$ & $83.8(78.0,88.6)$ & $49.3(43.9,54.7)$ & $63.7(53.0,73.6)$ & $0.543(0.506,0.581)$ \\
$\geq 12$ & $14.5(10.3,19.7)$ & $92.2(87.6,95.5)$ & $48.5(43.4,53.6)$ & $68.0(53.3,80.5)$ & $0.533(0.504,0.563)$ \\
$\geq 13$ & $9.83(6.33,14.4)$ & $93.6(89.3,96.6)$ & $47.5(42.5,52.5)$ & $63.9(46.2,79.2)$ & $0.517(0.492,0.543)$ \\
\hline
\end{tabular}

Nevertheless, despite the promising result of the NEWS, further prospective multicenter studies are required for its validation. Sepsis is a dynamic disease, and any tool relying on a single reading of physiological parameters might have a limited accuracy for predicting deterioration. In addition, the prognosis of sepsis is affected by age, comorbidities, and source of the infection. Therefore, designing a special sepsis tool that integrates clinical risk factors with the physiological parameters used in the NEWS may provide greater specificity. Adding point-of-care lactate measurements as an adjunct might be an added value.

Our study has limitations that we acknowledge. Being a single-center study with a small sample size may affect the generalizability of these results. Additionally, we did not assess patients on beta-blockers or calcium-channel blockers in a subgroup analysis, as these drugs might affect heart rate and blood pressure response, which may affect the NEWS. Furthermore, lactate results were based on the initial result of venous blood analysis and not from the arterial blood samples. Given the fact that clinical life relies on venous lactate, this factor might affect the internal validity and not the external validity of the study.

\section{Conclusion}

Our study confirms the growing evidence that suggests the NEWS is a sensitive screening tool in the ED for predicting sepsis-related outcomes. We have also demonstrated that this remains so when used in a triage setting conducted by nurses. However, it was not sufficiently specific, and further prospective multicenter studies are required for its validation in that role. We recommend designing a special sepsis tool that integrates clinical risk factors with the physiological parameters used in the NEWS to enhance specificity.

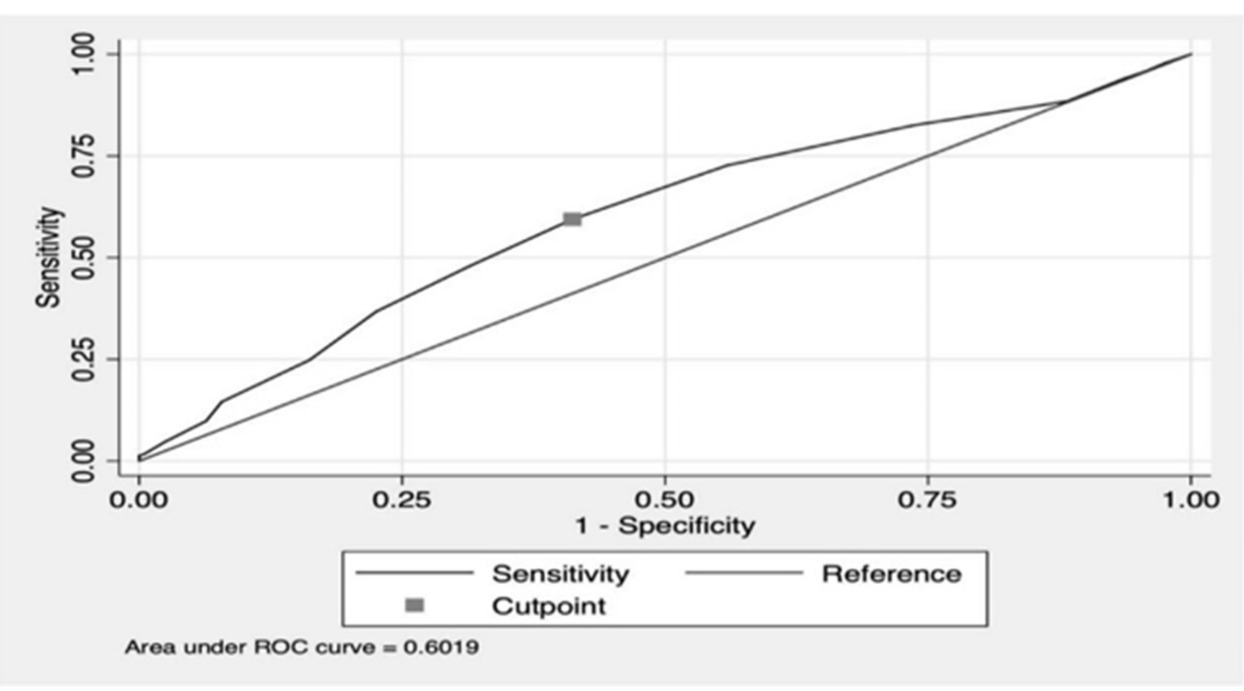

Figure 5 NEWS as a predictor for combined worse outcome (ICU admission or mortality). 


\section{Abbreviations}

AUC, area under the curve; ED, emergency department; $\mathrm{ICU}$, intensive care unit; IQR, interquartile range; KSMC, King Saud Medical City; NEWS, National Early Warning Score; NPV, negative predictive value; PPV, positive predictive value; qSOFA, quick Sepsis-related organ failure assessment; ROC, receiver operating characteristic; SIRS, systemic inflammatory response.

\section{Acknowledgment}

Deep and sincere thanks extend to sepsis project team members in KSMC, Mr. Bashar Abujudeh, Mr. Emad Alkhatib, Ms. Maryam Thekrallah, Mr. Abdulaziz Elsaeed, and last but not least to the secretary Ms. Vivian Darlucio. Besides, we would like to thank All Emergency Department personnel of KSMC for the great support and facilitation especially triage nurses who were doing sepsis screening in our sepsis improvement project.

\section{Author Contributions}

Writing the article by Saqer Althunayyan, Abdulrahman Alqahtani, Badar Alotaibi, Marwa Ahmed, Isam Osman, Fatma Bashraheel, and Adil Kakpuri, each of the authors contributed equally in writing the article. Khaled Alenazi, Mohammed Arafat, Abdulmajeed Al-Mutairi collected the data and arbitrated cases of discrepancy or missing data and performed statistical analysis. Abdulaziz Almutary, Faisal Almazroua, and Abdulaziz Alanazi revised the article and prepared it according to the guidelines of the journal. Conceptualization, by Saqer Althunayyan and Abdulaziz Almutary and Faisal Almazroua; methodology, Fatma Bashraheel and Abdulrahman Alqahtani and Saqer Althunayyan; software, Abdulaziz Alanazi. Abdulmajeed Al-Mutairi validation, Mohammed Arafat and Isam Osman; formal analysis, Marwa Ahmed and Adil Kakpuri and Abdulrahman Alqahtani; investigation, Badar Alotaibi and Marwa Ahmed and Isam Osman; resources, Adil Kakpuri and Khaled Alenazi; data curation, Mohammed Arafat; writing - original draft preparation, Abdulmajeed Al-Mutairi and Fatma Bashraheel; writing — review and editing, Abdulaziz Almutary and Khaled Alenazi; visualization, Abdulaziz Alanazi; supervision, Faisal Almazroua; project administration, Badar Alotaibi. All authors made substantial contributions in drafting the article or revising it critically for important intellectual content, agreed to submit to the current journal, gave final approval of the version to be published, and agreed to be accountable for all aspects of the work.

\section{Disclosure}

The authors have no conflict of interest in this work.

\section{References}

1. Baharoon S, Telmesani A, Tamim H, et al. Community- versus nosocomial-acquired severe sepsis and septic shock in patients admitted to a tertiary intensive care in Saudi Arabia, etiology and outcome. J Infect Public Health. 2015;8(5):418-424. doi:10.1016/j.jiph.2014.12.003

2. Whittaker SA, Mikkelsen ME, Gaieski DF, Koshy S, Kean C, Fuchs BD. Severe sepsis cohorts derived from claims-based strategies appear to be biased toward a more severely ill patient population. Crit Care Med. 2013;41(4):945-953. doi:10.1097/CCM.0b013e31827466f1

3. Martin GS, Mannino DM, Eaton S, Moss M. The epidemiology of sepsis in the United States from 1979 through 2000. $N$ Engl J Med. 2003;348(16):1546-1554. doi:10.1056/NEJMoa022139

4. Moore LJ, Jones SL, Kreiner LA, et al. Validation of a screening tool for the early identification of sepsis. J Trauma. 2009;66 (6):1539-1547. doi:10.1097/TA.0b013e3181a3ac4b

5. Nguyen HB, Corbett SW, Steele R, et al. Implementation of a bundle of quality indicators for the early management of severe sepsis and septic shock is associated with decreased mortality. Crit Care Med. 2007;35(4):1105-1112. doi:10.1097/01.CCM.0000259463.33848.3D

6. Jones SL, Ashton CM, Kiehne L, et al. Reductions in sepsis mortality and costs after design and implementation of a nurse-based early recognition and response program. Jt Comm J Qual Patient Saf. 2015;41(11):483-491.

7. Levy MM, Evans LE, Rhodes A. The surviving sepsis campaign bundle: 2018 update. Intensive Care Med. 2018;44(6):925-928. doi:10.1007/s00134-018-5085-0

8. Chamberlain DJ, Willis E, Clark R, Brideson G. Identification of the severe sepsis patient at triage: a prospective analysis of the Australasian triage scale. Emerg Med J. 2015;32(9):690-697. doi:10.1136/emermed-2014-203937

9. Alam N, Doerga KB, Hussain T, et al. Epidemiology, recognition and documentation of sepsis in the pre-hospital setting and associated clinical outcomes: a prospective multicenter study. Acute Med. 2016;15 (4): $168-175$

10. Smyth MA, Brace-McDonnell SJ, Perkins GD. Identification of adults with sepsis in the prehospital environment: a systematic review. $B M J$ Open. 2016;6(8):e011218. doi:10.1136/bmjopen-2016-011218

11. Giamarellos-Bourboulis EJ, Tsaganos T, Tsangaris I, et al. Validation of the new sepsis-3 definitions: proposal for improvement in early risk identification. Clin Microbiol Infect. 2017;23(2):104-109.

12. Tusgul S, Carron PN, Yersin B, Calandra T, Dami F. Low sensitivity of qSOFA, SIRS criteria and sepsis definition to identify infected patients at risk of complication in the prehospital setting and at the emergency department triage. Scand J Trauma Resusc Emerg Med. 2017;25(1):108. doi:10.1186/s13049-017-0449-y

13. Brabrand M, Havshøj U, Graham CA. Validation of the qSOFA score for identification of septic patients: a retrospective study. Eur J Intern Med. 2016;36:e35-e6. doi:10.1016/j.ejim.2016.09.004

14. Alam N, Vegting IL, Houben E, et al. Exploring the performance of the National Early Warning Score (NEWS) in a European emergency department. Resuscitation. 2015;90:111-115. doi:10.1016/j. resuscitation.2015.02.011

15. Corfield AR, Lees F, Zealley I, et al. Utility of a single early warning score in patients with sepsis in the emergency department. Emerg Med J. 2014;31(6):482-487. doi:10.1136/ emermed-2012-202186

16. Brink A, Alsma J, Verdonschot R, et al. Predicting mortality in patients with suspected sepsis at the emergency department; A retrospective cohort study comparing qSOFA, SIRS and national early warning score. PLoS One. 2019;14(1):e0211133. doi:10.1371/ journal.pone. 0211133 
17. Churpek MM, Snyder A, Han X, et al. Quick sepsis-related organ failure assessment, systemic inflammatory response syndrome, and early warning scores for detecting clinical deterioration in infected patients outside the intensive care unit. Am J Respir Crit Care Med. 2017;195(7):906-911. doi:10.1164/rccm.201604-0854OC

18. Shetty AL, Thompson K, Byth K, et al. Serum lactate cut-offs as a risk stratification tool for in-hospital adverse outcomes in emergency department patients screened for suspected sepsis. BMJ Open. 2018;8(1):e015492. doi:10.1136/bmjopen-2016-015492

19. Filho RR, Rocha LL, Corrêa TD, Pessoa CM, Colombo G, Assuncao MS. Blood lactate levels cutoff and mortality prediction in sepsis-time for a reappraisal? A retrospective cohort study. Shock. 2016;46(5):480-485. doi:10.1097/SHK.0000000000000667

20. Goulden R, Hoyle MC, Monis J, et al. qSOFA, SIRS and NEWS for predicting inhospital mortality and ICU admission in emergency admissions treated as sepsis. Emerg Med J. 2018;35(6):345-349. doi:10.1136/emermed-2017-207120
21. Loritz M, Busch HJ, Helbing T, Fink K. Prospective evaluation of the quick SOFA score as a screening for sepsis in the emergency department. Intern Emerg Med. 2020;15(4):685-693.

22. Hwang SY, Jo IJ, Lee SU, et al. Low accuracy of positive qSOFA criteria for predicting 28-day mortality in critically ill septic patients during the early period after emergency department presentation. Ann Emerg Med. 2018;71(1):1-9.e2. doi:10.1016/j.annemergmed.2017.05.022

23. van der Woude SW, van Doormaal FF, Hutten BA, Nellen FJ, Holleman F. Classifying sepsis patients in the emergency department using SIRS, qSOFA or MEWS. Neth J Med. 2018;76(4):158-166.
Infection and Drug Resistance

\section{Publish your work in this journal}

Infection and Drug Resistance is an international, peer-reviewed openaccess journal that focuses on the optimal treatment of infection (bacterial, fungal and viral) and the development and institution of preventive strategies to minimize the development and spread of resistance. The journal is specifically concerned with the epidemiology of
Dovepress

antibiotic resistance and the mechanisms of resistance development and diffusion in both hospitals and the community. The manuscript management system is completely online and includes a very quick and fair peerreview system, which is all easy to use. Visit http://www.dovepress.com/ testimonials.php to read real quotes from published authors. 